\title{
Pharmacogenetics and the treatment of cardiovascular disease
}

Patients with cardiovascular disease, like other patient groups, vary in their response to drugs. This is true both in terms of the response of their disease to treatment and their ability to tolerate medication. Several factors contribute to this variability, including differences in body mass, co-existent renal disease, concomitant drug treatment, and the heterogeneous nature of the disease. The Human Genome Project and the general focus of clinical science on genetic determinants of disease has increased interest in the role of genetic factors in determining an individual's response to drugs. Pharmacogenetics is a growing discipline concerned with the effect of genetic variation on the response to drugs and the extent to which drug treatment can be tailored to the individual according to their genetic makeup.

\section{Genetic polymorphism}

The idea that the dose of a drug should be adjusted according to the patient's genotype is not new. It is well recognised that polymorphism in genes encoding P450 enzymes, $\mathrm{N}$-acetyltransferase, and other key enzymes of drug metabolism are responsible for major inter-individual differences in blood concentrations of certain drugs. Clinically, poor metabolisers may be slow to activate and respond inadequately to some pro-drugs, or exhibit reduced clearance and increased effects from some pharmacologically active agents, particularly when the latter have a steep dose-response curve or a narrow therapeutic index. For example, the principle route of metabolism of flecainide and propafenone is via cytochrome P450 2D6 and multiple alleles have been identified that impair the activity of the enzyme. Patients with poor CYP450 2D6 activity demonstrate higher blood concentrations of flecainide and propafenone and have more side effects for a given dose than those with normal enzyme activity. ${ }^{12}$ Awareness of a patient's genotype can protect that patient from certain drugs or their prescription in dangerous doses. Pharmacogenetics extends this approach of tailoring drug development and treatment beyond factors that regulate drug metabolism to other genetically determined variables that may influence the response of a patient to a given drug.

Some tentative progress has been made in this direction. Several genetic polymorphisms have been identified that appear to influence the response to pravastatin. ${ }^{3}$ For example, the Taq1B polymorphism of cholesteryl ester transfer protein (CETP), which has a key role in the metabolism of high density lipoprotein, has been reported to show a dose dependent correlation with severity of coronary atherosclerosis and predict the response to treatment with pravastatin; patients with the B1B1 genotype (homozygous for the restriction site for Taq1) demonstrated more severe disease and responded better to the statin than patients with the B2B2 genotype (homozygous for the absence of the restriction site), with the B1B2 genotype intermediate. ${ }^{4}$
Patients with heart failure harbouring the 1 le $164 \beta_{2}$ adrenergic receptor polymorphism, which significantly alters the function of the receptor (it reduces the binding affinity of the receptor for catecholamines and certain $\beta$ receptor antagonists, decreases basal and stimulated adenyl cyclase activity and agonist stimulated sequestration of the receptor), demonstrate a more rapid progression of their disease. ${ }^{5}$ This variable should therefore be considered when patients are randomised in clinical trials of new treatments.

\section{SNP Consortium}

At first sight the pharmaceutical industry might be expected to have little interest in this extended application of pharmacogenetics as any targeting of treatment will segment the market. In reality, the industry sees benefits from this approach in terms of drug development and market access. Genotyping offers companies the potential to select appropriate patients for clinical studies of new drugs, thereby reducing the numbers required in trials, speeding up drug development, and reducing costs. It may even rescue some drugs that appear to be effective only in a specific subset of the population. By identifying patients most likely to respond or by eliminating those most at risk of adverse events it will be easier to establish value for a particular medicine, leading to more rapid acceptance and clinical use.

Pharmaceutical companies are already genotyping subjects participating in clinical trials for candidate genes that may influence response to the test compound(s). Recently, a consortium of major pharmaceutical companies, the Wellcome Trust, and several genome centres have organised the SNP Consortium (http://snp.cshl.org), a non-profit making company that aims to produce a high density, ordered map of 300000 single nucleotide polymorphism (SNP) by April 2001. SNPs occur on average once in every 1000 bases of the three billion bases in the human genome. The pharmaceutical companies hope to use the SNP map to construct polymorphism profiles associated with drug response and adverse effects in clinical trials, but the map will also be available to academics (http://www.ncbi.nlm.nih.gov/SNP).

\section{Practical issues}

Several practical issues-technological, biological, and ethical-need to be addressed for pharmacogenetics to have a real impact. Consider a large phase 2 study of 500 patients, divided into responders and non-responders. To characterise the genetic profiles of each group requires processing over 200000 allelic variants for each individual within a reasonable time scale and at an acceptable cost. Performed in duplicate, this could translate into 400000 data points per individual or 200000000 assays; over four weeks ( 20 working days), this would mean about 10 million assays per day. This is a tall order but the pharmaceutical 
industry and biotechnology groups are exploring the methodology required to meet the task. It is anticipated that the technology will become operational in the next 3-5 years with the effects felt on drug development over the next decade.

It is not envisaged that doctors will need to fully genotype their patients in routine clinical practice. Abbreviated SNP profiles characteristic of that associated with a "genetic responder" could be made available for each drug. These profiles will enable patients to be screened using comparatively less expensive high throughput, readout systems, such as DNA microarrays ("chips") or lower cost encoded bead based methods currently in development. ${ }^{6}$

SNP profiles will need to be validated and approved by the regulatory authorities. Establishing a secure relation between genotype and phenotype can be difficult. There are examples of monogenic disorders where having "the genotype" does not necessarily mean that the person will exhibit the disease. ${ }^{7}$ Some authors doubt the statistical power of conventional multicentre clinical trials to demonstrate linkage between genotype and phenotype. It has been pointed out that at the $1 \%$ significance level $(\mathrm{p}<0.01)$, an analysis of 1-500 000 SNPs will produce 1-5000 false positive results. ${ }^{8}$ To achieve approval, genetic analysis needs to be incorporated prospectively in the design of pivotal trials. It is suggested that investigators should concentrate on those genes that are most likely to have significant effects on drug metabolism or pathways of drug action than randomly selected markers. This approach, together with association studies based on haplotypes, may reduce the complexity of study design while improving the sensitivity of the analysis. Recognition of these difficulties has lead to the emergence of departments of bioinformatics to deal specifically with the arguments concerning the appropriate collection and statistical analysis of genotype data and its applicability to different ethnic groups.

The use of genetic information is always a sensitive issue. Nonetheless, data that facilitate the safe and effective development and prescribing of medicines should be welcomed. A partial solution is to provide an abbreviated "genetic profile chip" for each drug that is anonymous with respect to its composition; it is used simply to inform on the probability that a patient is likely to respond to or develop adverse effects from that drug. This would reduce the potential for collecting genetic information and "insurance scares" such as those that accompany current discussions of defined monogenic disease mutations. The futuristic notion that patients will be issued with "genetic profile cards" that inform physicians which drugs they should be taking and which to avoid is limited currently not only by technology but also by the need for society to decide on how it is going to cope with the ethical concerns of susceptibility genetics.

It is easy to become mesmerised by advances in genetics. It is important not to lose touch with established principles. Not all drug responses are determined by inheritance and not all genetically determined responses will be easy to measure. Whatever the contribution of pharmacogenetics to future prescribing, data on a patient's genotype should not be used in isolation but in conjunction with other well established medical and ethical guidelines as part of the treatment decision making process.

MARTIN R WILKINS ALLEN D ROSES*

Section on Clinical Pharmacology,

C PIERS CLIFFORD

Imperial College School of Medicine,

Hammersmith Hospital

London W12 ONN, UK

${ }^{\star}$ Glaxo Wellcome Research and Development,

Five Moor Drive, PO Box 13398

Research Triangle Park,

North Carolina 27709-3398, USA

Correspondence to: Professor Wilkins

m.wilkins@ic.ac.uk

1 Mikus G, Gross AS, Beckmann J, et al. The influence of the sparteine/debrisoquine phenotype on the disposition of flecanide. Clin Pharmacol Ther 1989;45:562-7.

2 Siddoway LA, Thompson KA, McAllister CB, et al. Polymorphism of propafenone metabolism and disposition in man, clinical and pharmacokinetic consequences. Circulation 1987;75:785-91.

3 Jukema JW. Matching treatment to the genetic basis of (lipid) disorder in patients with coronary artery disease. Heart 1999;82:126-7.

4 Kuivenhoven JA, Jukema JW, Zwinderman AH, et al. The role of a common variant of the cholesteryl ester transfer protein gene in the progression of coronary atherosclerosis. N Engl f Med 1998;338:86-93.

5 Liggett SB, Wagoner LE, Craft LL, et al. The Ile 164 beta $_{2}$-adrenergic receptor polymorphism adversely affects the outcome of congestive heart failure. f Clin Invest 1998;102:1534-9.

6 Lander E. Array of hope. Nature Genet 1999;21:3-4.

7 Fananapazir L, Epstein ND. Genotype-phenotype correlations in hypertrophic cardiomyopathy. Circulation 1994;89:22-32.

8 Ledley FD. Can pharmacogenetics make a difference in drug development? Nature Biotech 1999;17:731. 